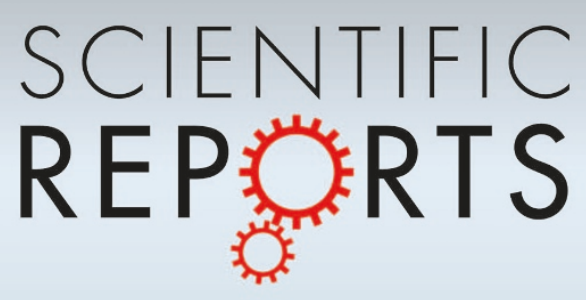

OPEN

SUBJECT AREAS:

SUPERCONDUCTING PROPERTIES AND

MATERIALS

SUPERCONDUCTING DEVICES

NANOWIRES

Received

24 September 2014

Accepted

13 January 2015

Published

9 February 2015

Correspondence and requests for materials should be addressed to

A.G. (agalda@anl. gov)

\title{
Resonant tunneling of fluctuation Cooper pairs
}

\author{
Alexey Galda', A. S. Mel'nikov ${ }^{2,3}$ \& V. M. Vinokur'
}

\begin{abstract}
${ }^{1}$ Materials Science Division, Argonne National Laboratory, Argonne, Illinois 60439, USA, ${ }^{2}$ Institute for Physics of Microstructures, Russian Academy of Sciences, 603950 Nizhny Novgorod, GSP-105, Russia, ${ }^{3}$ Lobachevsky State University of Nizhny Novgorod, 23 Prospekt Gagarina, 603950, Nizhny Novgorod, Russia.
\end{abstract}

Superconducting fluctuations have proved to be an irreplaceable source of information about microscopic and macroscopic material parameters that could be inferred from the experiment. According to common wisdom, the effect of thermodynamic fluctuations in the vicinity of the superconducting transition temperature, $T_{\mathcal{O}}$ is to round off all of the sharp corners and discontinuities, which otherwise would have been expected to occur at $T_{c}$. Here we report the current spikes due to radiation-induced resonant tunneling of fluctuation Cooper pairs between two superconductors which grow even sharper and more pronounced upon approach to $T_{c}$. This striking effect offers an unprecedented tool for direct measurements of fluctuation Cooper pair lifetime, which is key to our understanding of the fluctuation regime, most notably to nature of the pseudogap state in high-temperature superconductors. Our finding marks a radical departure from the conventional view of superconducting fluctuations as a blurring and rounding phenomenon.

$\mathrm{D}$ oes every of the fascinating manifestations of the coherent nature of the Cooper pairs below the superconducting critical temperature have its counterpart in the fluctuation regime above $T_{c}$ ? Fluctuation paraconductivity ${ }^{1,2}$, diamagnetism ${ }^{3,4}$, Hall and Nernst effects above $T_{c}^{4-8}$, to name a few (see Ref. 9 and references therein), all come with an affirmative. A fluctuation counterpart of Shapiro spikes ${ }^{10}$, resonant jumps in dc current in biased Josephson junctions in response to external ac voltage, foreseen by Kulik ${ }^{11}$, appears to be of special interest. We develop a theory of fluctuation Shapiro resonances, which we realize in the regime of passing the dc current through the barrier between two superconductors taken above the critical temperature, and find that, contrary to its counterpart below $T_{c}$, this resonant effect is highly sensitive to the temperature dependence of the lifetime of superconducting fluctuations. We demonstrate that the emergence of a new characteristic time above $T_{c}$, the lifetime of fluctuation Cooper pairs $\tau_{G L} \sim \hbar / k_{B}\left(T-T_{C}\right)$, where $\hbar$ and $k_{B}$ are the Planck and Boltzmann constants, respectively, leads to new distinct resonant features in the current-voltage characteristics, which offer a direct access to high-precision measurements of $\tau_{G L}$.

Shapiro resonances that result from tuning or synchronization between the intrinsic Josephson frequency and that of the external source are one of the profound manifestations of the Josephson physics. Below $T_{c}$ this effect provides the currently most accurate physical standards for the volt ${ }^{12}$. We propose a theory and a simple experimental verification of the effect of resonant tunneling of fluctuation Cooper pairs at temperatures above critical. This fluctuational analog of the inverse ac Josephson effect can be used to verify the origin of the pseudogap regime in high-temperature superconductors.

Above $T_{c}$ Shapiro resonances can be most conveniently realized in a biased superconductor-insulator-superconductor (SIS) junction with applied ac voltage, induced by external microwave radiation of frequency $\omega$, see Fig. 1. We let the total applied voltage across the junction be $V(t)=V_{0}+V_{1} \cos (\omega t)$. This produces equally separated jumps in $\mathrm{dc}$ current through the junction at the following values of the dc bias voltage: $V_{0}^{(n)}=n \hbar \omega / 2 e$, where $n$ is an integer, i.e. when Cooper pairs with charge $2 e$ pass across the junction. To derive the current-voltage $(I-V)$ characteristics in Gaussian fluctuation regime, we employ the linearized time-dependent GinzburgLandau (TDGL) theory containing the Langevin noise term with white noise Gaussian correlations. Linearized TDGL theory describes the system near but certainly outside the range of strong fluctuations, defined by the respective Ginzburg criterion ${ }^{9}, G i<\epsilon \ll 1$, where $\epsilon=\left(T-T_{c}\right) / T_{c}$ and $G i$ is the Ginzburg-Levanyuk number. The insulating barrier is placed at the plane $x=0$ and is supposed to be high enough, so that it accommodates most of the voltage drop. We neglect the spatial dependence of the electric scalar potential in the bulk. The width of the insulating layer (the $x$-dimension, see Fig. 1) is assumed to be small as compared to the Ginzburg-Landau 


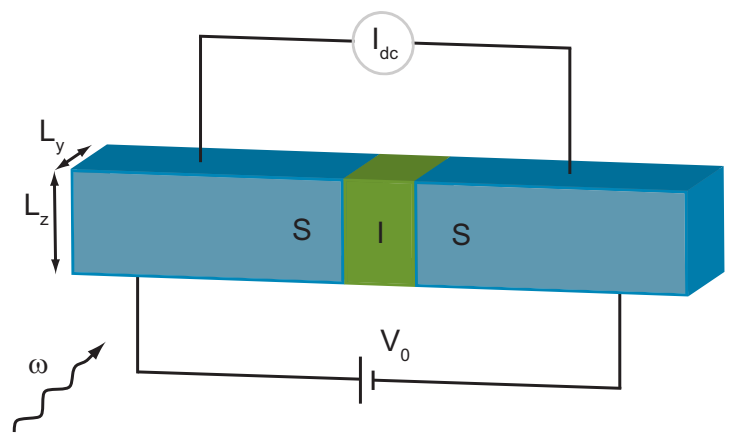

Figure 1 System setup. Proposed four-point measurement of the $I-V$ characteristics of an ac $+\mathrm{dc}$ voltage biased tunnel junction. Transition between $1 \mathrm{D}$ and $3 \mathrm{D}$ geometries can be achieved experimentally by varying temperature and lateral dimensions $L_{y}$ and $L_{z}$ of the insulating (I) layer sandwiched between two superconductors $(S)$.

coherence length $\xi(T)=\xi_{0} / \sqrt{\epsilon}$. The role of the barrier is to impose linear boundary conditions ${ }^{13}$ for the superconducting order parameter in the plane $x=0$, which give rise to the Josephson tunneling of Cooper pairs. In the Supplementary Information we provide the system of coupled TDGL differential equations for the order parameter on either side of the insulating barrier, together with the aforementioned boundary conditions. In the limit of weak tunneling current through a symmetric SIS junction, we solve the problem perturbatively and write the expression for the $\mathrm{dc}$ component of the tunneling current. The result is obtained in the second order in the barrier transparency, while the first order contribution vanishes. First, we consider a narrow one-dimensional (1D) junction in the temperature range where the lateral dimensions are much smaller than the Ginzburg-Landau coherence length, $L_{y}, L_{z} \ll \xi(T)$. In this case the junction carries a single transverse fluctuation mode. We then extend our approach to the two- and three-dimensional setups with multiple transverse modes. For the system to be in the $2 \mathrm{D}$ regime, the size of the junction must be such that $L_{y} \gg \xi(T) \gg L_{z}$. Similarly, if both cross-sectional dimensions of the junction much exceed the size of Cooper pairs, $L_{y}, L_{z} \gg \xi(T)$, the system is considered to be in the $3 \mathrm{D}$ regime. To calculate the total tunneling current we perform a summation over all transverse momenta in Fourier space. The detailed calculation is provided in the Supplementary Information.

\section{Results}

We first analyze the $I-V$ characteristics of a $1 D$ junction. Depending on the parameters of the system, we find that there are three main regimes: a) $\omega \tau_{\mathrm{GL}} \ll 1$, b) $\omega \tau_{\mathrm{GL}} \gg 1$ and c) $\omega \tau_{\mathrm{GL}} \sim 1$, where the characteristic timescale $\tau_{\mathrm{GL}}=\frac{\pi \hbar}{8 k_{B} T_{c} \epsilon}$ is the lifetime of Cooper pair fluctuations. In the first case, the frequency of the external ac source is low, so that temporal variations in voltage over the lifetime of Cooper pairs are negligible and the system is in the stationary regime. One can show (see Supplementary Information) that when only a positive constant voltage $V_{0}$ is applied to the junction, the tunneling current is a non-oscillating function of voltage, reaching its maximum at $V_{0 \max } \simeq \frac{5 \hbar}{2 e \tau_{\mathrm{GL}}}$ and decaying at large voltages. Therefore, when averaged over the period of the ac bias oscillations, the peak of the stationary $I-V$ characteristic $I\left(V_{0}\right)$ is observed at $V_{0 \text { max }}$. The second regime, $\omega \tau_{\mathrm{GL}} \gg 1$, is the primary focus of this work. It is characterized by the appearance of equidistant resonant Shapiro spikes on the $I-V$ curve at bias voltages $V_{0}^{(n)}$, see Fig. 2. The number of these resonant features is limited by the value of the ratio $2 e V_{1} / \hbar \omega$, with only one peak at the origin when $2 e V_{1} / \hbar \omega \ll 1$, see Supplementary Fig. S1. The

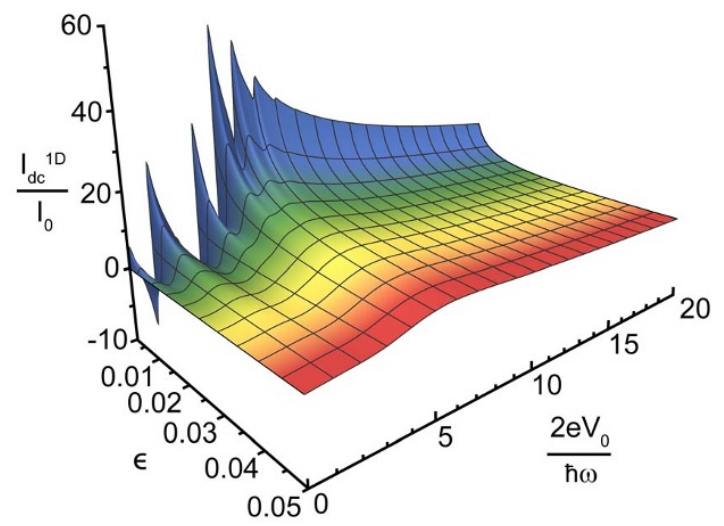

Figure $2 \mid$ Resonant Shapiro spikes in tunneling current of fluctuation Cooper pairs. Numerical simulation of $I-V$ characteristics as a function of reduced temperature $\epsilon$ and applied dc voltage $V_{0}$ for the $1 \mathrm{D}$ junction at temperatures above critical. Sharp linear spikes in tunneling current at resonant dc voltages $V_{0}^{(n)}$ are developed upon approaching $T_{c}$. The corresponding values of $\omega \tau_{\mathrm{GL}}$ are between 2 and 100, which illustrates a transition between the stationary and resonant regimes. Parameters used in the simulation: $2 e V_{1}=5 \hbar \omega, \pi \hbar \omega / 8 k_{B} T_{c}=0.1$, which experimentally corresponds to a junction with $T_{c}=10 \mathrm{~K}$ under microwave radiation of frequency $53 \mathrm{GHz}$ and ac voltage amplitude $V_{1}=0.33 \mathrm{mV}$.

resonances appear on the $I-V$ curve as linear spikes with the slope proportional to $\omega \tau_{\mathrm{GL}} / \epsilon$. We note a remarkable universality of the shape of the peaks at fixed $2 e V_{1} / \hbar \omega$ and $n$ : their height and width are equal to $c_{1} \tau_{\mathrm{GL}}$ and $c_{2} \tau_{\mathrm{GL}}^{-1}$, correspondingly, where $c_{1}$ and $c_{2}$ are some fixed numeric parameters independent of $\tau_{\mathrm{GL}}$. This unique property of Shapiro resonances above $T_{c}$ can serve as a convenient experimental tool for direct determination of the lifetime of fluctuation Cooper pairs. Finally, when $\omega \tau_{\mathrm{GL}} \sim 1$, one observes a smooth transition between the stationary and resonant regimes, which occurs when the slope of the $I-V$ curves around $V_{0}^{(n)}$ becomes comparable to the slope of the stationary $I-V$ characteristic. We note that external ac voltage can cause Cooper pair fluctuations to tunnel in the opposite direction to the applied dc bias at voltages just below the resonant values $V_{0}^{(n)}$ for low $n$.

In Fig. 3 we plot the $I-V$ characteristics for $1 \mathrm{D}$ and $3 \mathrm{D}$ setups, illustrating that the effect of resonant fluctuation Cooper pair tunneling should be observable across all effective dimensionalities of the SIS junction. The total current in the $3 \mathrm{D}$ case, depicted in Fig. 3b, is normalized by the characteristic number of fluctuation modes $n_{f}^{3 D}=L_{y} L_{z} /\left(2 \pi \xi_{0}\right)^{2}$. As can be seen from the expression for the current in $3 \mathrm{D}$ (see Methods), $\omega \tau_{\mathrm{GL}}$ is no longer a single parameter defining the shape of the resonances. Instead, the ratio $\hbar \omega / k_{B} T_{c}$ now determines how well pronounced the spikes around $V_{0}^{(n)}$ can be upon approaching the critical temperature, i.e. lowering $\epsilon$. In order to clearly observe sharp Shapiro resonances in the Gaussian fluctuation regime for a $3 \mathrm{D}$ junction, much higher values of $\hbar \omega / k_{B} T_{c}$ are required as compared to the $1 \mathrm{D}$ case, by an order of magnitude or more, assuming the same range of temperatures. This is due to the fact that the slope of Shapiro spikes in 3D is much less sensitive to $\epsilon$ as one approaches $T_{c}$. In fact, in close vicinity of each resonant value $V_{0}^{(n)}$ the tunneling current takes the following form:

$$
\langle I\rangle_{\mathrm{dc}} \propto J_{n}^{2}\left(\frac{2 e V_{1}}{\hbar \omega}\right) \omega \tau_{\mathrm{GL}} \epsilon^{\frac{d-3}{2}}\left(V_{0}-V_{0}^{(n)}\right),
$$

where $J_{n}$ is the Bessel function of the first kind and $d$ is the effective dimensionality of the junction. While for 1D junctions Shapiro peaks should be observable at frequencies of tens of $\mathrm{GHz}$ and just fractions of a Kelvin away from a typical critical temperature $T_{c} \sim 10 \mathrm{~K}$, in 

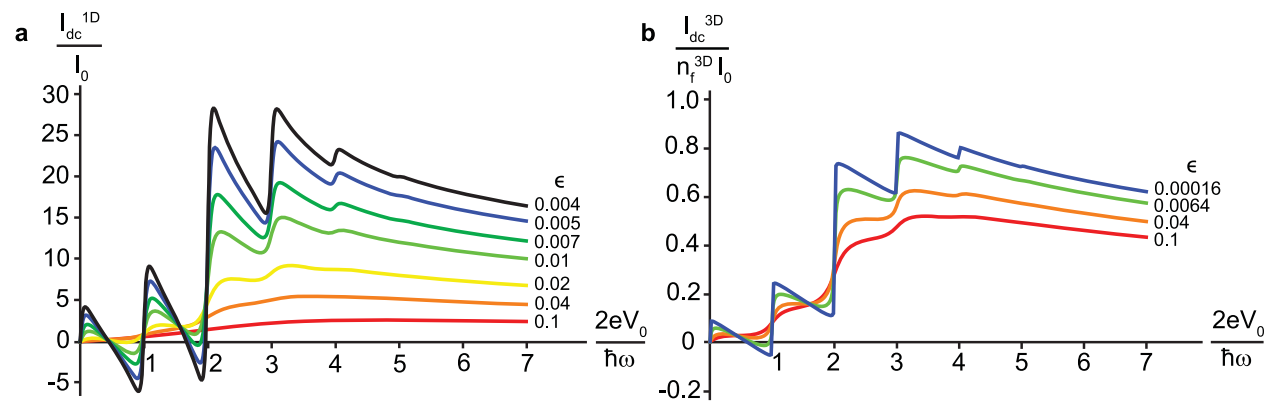

Figure $3 \mid I-V$ characteristics for $1 \mathrm{D}$ and 3D SIS junctions in fluctuation regime. (a) Tunneling current through the 1D junction develops resonant spikes at resonant values $V_{0}^{(n)}$, which grow sharper upon approaching $T_{c}$ due to increasing lifetime of fluctuation Cooper pairs. At higher dc bias voltages the current decreases monotonically as a function of $V_{0}$. System parameters used for the calculation: $2 e V_{1}=3 \hbar \omega, \pi \hbar \omega / 8 k_{B} T_{c}=0.2$. (b) Tunneling current in the $3 \mathrm{D}$ model. For the resonant spikes in the $3 \mathrm{D}$ setup to be visible, one requires higher ratios of $\hbar \omega / k_{B} T_{c}$ as compared to the $1 \mathrm{D}$ system. Parameters used: $2 e V_{1}=3 \hbar \omega, \pi \hbar \omega / 8 k_{B} T_{c}=2$, which is equivalent to $T_{c}=10 \mathrm{~K}$, microwave frequency of $1 \mathrm{THz}$ and $V_{1}=6.6 \mathrm{mV}$.

order to produce reasonably sharp resonant features in the $3 \mathrm{D}$ regime, one would need to use frequencies of at least hundreds of $\mathrm{GHz}$ - several $\mathrm{THz}$ and be able to measure temperature with almost millivolt precision.

To take into account thermal voltage fluctuations in the voltagebiased Josephson junction, we introduce an additional voltage noise term $v(t)$ in the applied voltage $V(t)$. The statistical properties of the voltage noise are defined by the following correlation function:

$$
\left\langle v(t) v\left(t^{\prime}\right)\right\rangle=2 k_{B} R T \delta\left(t-t^{\prime}\right),
$$

where $R$ is the tunneling resistance in normal state. The effect of voltage noise will manifests itself as an additional factor $\exp \left[\frac{2 i e}{\hbar} \int_{t_{1}}^{t} \mathrm{~d} t^{\prime} v\left(t^{\prime}\right)\right]$ under the integral in the expression for the $\mathrm{dc}$ current, equation (4) in Methods. Averaging over noise can be performed with the help of the cumulant expansion to yield

$$
\langle I\rangle_{\mathrm{dc}}^{1 D}=\frac{I_{0}}{\epsilon} \mathcal{F}\left(V_{0}, V_{1} ; \omega \tau_{\mathrm{GL}}, \Lambda\right),
$$

where $\Lambda \equiv 4 k_{B} e^{2} R T \tau_{G L} / \hbar^{2}$ determines the magnitude of thermal voltage noise and function $\mathcal{F}$ is given in the Methods section.

Numerical analysis based on equation (8) in Methods for a typical junction with normal state resistance $R \sim 10 \Omega$ shows that the effects of voltage fluctuations only become noticeable at temperatures as close to critical as $\epsilon \lesssim 4 \times 10^{-3}$, see Fig. 4 . Even when the noise level parameter $\Lambda \lesssim 1$, the spikes on the $I-V$ characteristics become only mildly smeared, with the features completely vanishing at $\Lambda \gtrsim 10$. Therefore, the predicted effect of Shapiro spikes due to superconducting fluctuations is robust against thermal voltage noise and should be experimentally observable in a wide range of temperatures.

\section{Discussion}

We have developed a simple phenomenological theory of the resonant transport of fluctuation Cooper pairs through the tunneling barrier under the influence of external radiation. The above consideration uncovers a novel fluctuation phenomenon in superconducting junctions, which is reminiscent to the inverse ac Josephson effect.

Extension of Shapiro resonances onto the $T>T_{c}$ range, where they are caused by resonant tunneling of the fluctuation Cooper pairs, opens yet one more area of applications of this phenomenon. Since tuning frequency ensures the best accuracy as compared to variation of other controlling parameters, the observation of the Shapiro spikes in the fluctuation regime can provide unprecedented precision measurements of the lifetime of fluctuation Cooper pairs. This precision is restricted only by the intrinsic noise of the system. Note that if we switch from the voltage-biased setup, which we discuss in our work, to the current-biased configuration, the predicted current spikes would transform into a series of plateaus positioned at voltages $n \hbar \omega / 2 e$, where $n$ is an integer. It is the length of these plateaus that would be equal to the amplitude of the corresponding resonant spike in the voltage-biased setup.

The appearance of such resonant features on the $I-V$ curves of high-temperature superconducting junctions could clarify the relevance of superconducting fluctuations in the pseudogap regime. An alternative way to probe fluctuating Cooper pairs in the pseudogap state is through the measurements of the Josephson current noise spectrum, as suggested in Refs. 14, 15.

\section{Methods}

To reveal the dependence of the $I-V$ curve on the system's dimensionality, one can evaluate the current for different transverse dimensions $L_{y}, L_{z}$ of the superconducting electrode cross section. The dc tunneling current for a thin wire with $L_{y}, L_{z} \lesssim \xi(T)$, averaged over superconducting fluctuations, is given by

$$
\langle I\rangle_{\mathrm{dc}}^{1 D}=\frac{I_{0}}{\epsilon} \sum_{n=-\infty}^{\infty} J_{n}^{2}\left(\frac{2 e V_{1}}{\hbar \omega}\right) \int_{0}^{\infty} \mathrm{d} \tau \sin \left[\left(\frac{2 e V_{0}}{\hbar \omega}-n\right) \omega \tau_{\mathrm{GL}} \tau\right] \frac{\mathrm{e}^{-\tau}}{\sqrt{\tau}} \operatorname{erfc}(\sqrt{\tau}),
$$

where $I_{0}=8 k_{B} \beta^{2} e T \xi_{0}^{2} / \hbar \sqrt{\pi}, \xi_{0}^{-2}=4 k_{B} m \alpha T_{c} / \hbar^{2}, \alpha$ is the phenomenological Ginzburg-Landau constant and $\beta$ is the parameter characterizing boundary conditions at the S-I boundary (see Supplementary Information). The dimensionless time $\tau$ is measured in units of $\tau_{\mathrm{GL}}$. Note that the first non-vanishing contribution occurs in the second order in the barrier transparency $\left(\propto \beta^{2}\right)$. To calculate the total tunneling current across junctions of higher dimensionality, we need to additionally sum over transverse momenta $\mathbf{p}_{\|}$in Fourier space (see Supplementary Information for more details). For a junction with $L_{y} \gg \xi(T) \gg L_{z}$ we can replace the summation over discrete momenta with integration:

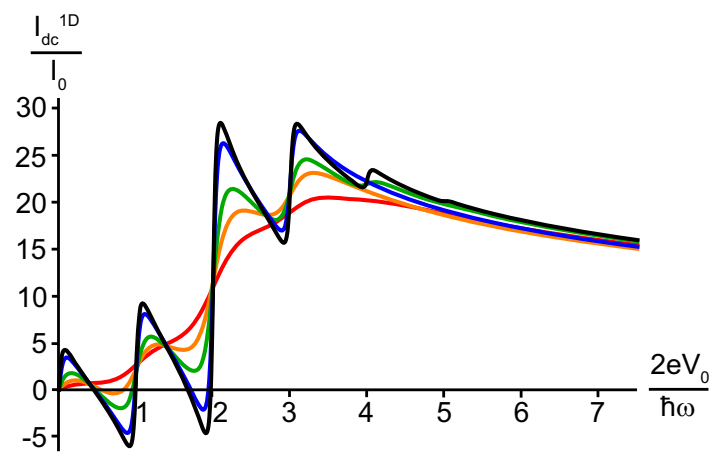

Figure $4 \mid$ Smearing of resonant tunneling features by thermal voltage noise. Normalized tunneling current of fluctuation Cooper pairs as a function of $V_{0}$ for various noise levels, $\Lambda=0$ (black), 1(blue), 5(green), 10 (orange), 20(red), where we used the same system parameters as in Fig. 3a. White thermal voltage noise produces very little visible effect on the $I-V$ characteristics of $1 \mathrm{D}$ junctions at $\Lambda \lesssim 1$, while higher values of the noise level parameter $\Lambda$ cause smearing of the Shapiro resonances. 
$\langle I\rangle_{\mathrm{dc}}^{2 D}=n_{f}^{2 D} I_{0} \sum_{n=-\infty}^{\infty} J_{n}^{2}\left(\frac{2 e V_{1}}{\hbar \omega}\right) \int_{0}^{\infty} \mathrm{d} \tau \frac{\mathrm{e}^{-\tau}}{\sqrt{\tau}} \operatorname{erfc}(\sqrt{\tau}) \int_{0}^{1} \frac{\mathrm{d} \rho}{\epsilon+\rho^{2}} \sin \left[\left(\frac{2 e V_{0}}{\hbar \omega}-n\right)\left(\frac{\pi \hbar \omega}{8 k_{B} T_{c}}\right) \frac{\tau}{\epsilon+\rho^{2}}\right],(5)$

where $n_{f}^{2 D}=L_{y} / 2 \pi \xi_{0}$ is the characteristic number of fluctuation modes. By analogy, in the $3 \mathrm{D}$ limit, $L_{y}, L_{z} \gg \xi(T)$, one obtains:

$\langle I\rangle_{\mathrm{dc}}^{3 D}=n_{f}^{3 D} I_{0} \sum_{n=-\infty}^{\infty} J_{n}^{2}\left(\frac{2 e V_{1}}{\hbar \omega}\right) \int_{0}^{\infty} \mathrm{d} \tau \frac{\mathrm{e}^{-\tau}}{\sqrt{\tau}} \operatorname{erfc}(\sqrt{\tau}) \int_{0}^{1} \frac{\mathrm{d} \rho}{\epsilon+\rho} \sin \left[\left(\frac{2 e V_{0}}{\hbar \omega}-n\right)\left(\frac{\pi \hbar \omega}{8 k_{B} T_{c}}\right) \frac{\tau}{\epsilon+\rho}\right]$,

with $n_{f}^{3 D}=L_{y} L_{z} /\left(2 \pi \xi_{0}\right)^{2}$. The integral over the $\rho$ variable in the $d$-dimensional case, where $\rho=\left(\xi_{0}\left|\mathbf{p}_{\|}\right| / \hbar\right)^{d-1}$, has an upper cut-off of the order unity, since the transverse momenta of the fluctuating wave functions within the Ginzburg-Landau theory must be smaller than the inverse coherence length, $\left|\mathbf{p}_{\|}\right|<\hbar \xi_{0}^{-1}$. It is important to note that the transition from a summation over quantized transverse momenta to the integration over $\rho$ requires that the relevant lateral dimensions of the junction considerably exceed the effective size of Cooper pairs, $L_{y, z} \gg \xi(T)$. Otherwise, at temperatures close enough to $T_{c}$ when $L_{y, z} \sim \xi(T)$ (if still within the region of applicability of our approach) a careful summation over discrete transverse modes must be performed. When further approaching the superconducting transition, the size of Cooper pairs grows and the effective dimensionality of the junction can decrease.

Effect of thermal voltage noise. One can carry out the averaging over thermal voltage noise via the cumulant expansion:

$$
\left\langle e^{\frac{2 i e}{\hbar} \int_{t_{1}}^{t} \mathrm{~d} t^{\prime} v\left(t^{\prime}\right)}\right\rangle=e^{-\frac{2 e^{2}}{\hbar^{2}}\left\langle\left[\int_{t_{1}}^{t} \mathrm{~d} t^{\prime} v\left(t^{\prime}\right)\right]^{2}\right\rangle}=e^{-\frac{4 k_{B} e^{2}}{\hbar^{2}} R T\left(t-t_{1}\right)} .
$$

Using this identity, one finds the following expression for the function $\mathcal{F}$ from equation (3) of the main text, which determines the tunneling current per one fluctuation mode and accounts for thermal voltage noise:

$\mathcal{F}\left(V_{0}, V_{1} ; \omega \tau_{\mathrm{GL}}, \Lambda\right)=\sum_{n=-\infty}^{\infty} J_{n}^{2}\left(\frac{2 e V_{1}}{\hbar \omega}\right) \int_{0}^{\infty} \mathrm{d} \tau \sin \left[\left(\frac{2 e V_{0}}{\hbar \omega}-n\right) \omega \tau_{\mathrm{GL}} \tau\right] \frac{e^{-(1+\Lambda) \tau}}{\sqrt{\tau}} \operatorname{erfc}(\sqrt{\tau}) .(8)$

1. Aslamasov, L. G. \& Larkin, A. I. The influence of fluctuation pairing of electrons on the conductivity of normal metal. Phys. Lett. A 26, 6 238-239 (1968).

2. Friedmann, T. A., Rice, J. P., Giapintzakis, J. \& Ginsberg, D. M. In-plane paraconductivity in a single crystal of superconducting $\mathrm{YBa}_{2} \mathrm{Cu}_{3} \mathrm{O}_{7-x}$. Phys. Rev. B 39, 74258 (1989).

3. Skocpol, W. J. \& Tinkham, M. Fluctuations near superconducting phase transitions. Reports on Progress in Physics 38, 91049 (1975).

4. Li, L. et al. Diamagnetism and Cooper pairing above $T_{c}$ in cuprates. Phys. Rev. B 81, 5054510 (2010).
5. Ullah, S. \& Dorsey, A. T. Effect of fluctuations on the transport properties of typeII superconductors in a magnetic field. Phys. Rev. B 44, 1262 (1991).

6. Serbyn, M. N., Skvortsov, M. A., Varlamov, A. A. \& Galitski, V. Giant Nernst effect due to fluctuating Cooper pairs in superconductors. Phys. Rev. Lett. 102, 6067001 (2009).

7. Xu, Z. A., Ong, N. P., Wang, Y., Kakeshita, T. \& Uchida, S. Vortex-like excitations and the onset of superconducting phase fluctuation in underdoped $\mathrm{La}_{2-x} \mathrm{Sr}_{x} \mathrm{CuO}_{4}$. Nature 406, 6795 486-488 (2000).

8. Wang, Y. et al. Onset of the vortexlike Nernst signal above Tc in $\mathrm{La}_{2-x} \mathrm{Sr}_{x} \mathrm{CuO}_{4}$ and $\mathrm{Bi}_{2} \mathrm{Sr}_{2-y} \mathrm{La}_{y} \mathrm{CuO}_{6}$. Phys. Rev. B 64, 22224519 (2001).

9. Larkin, A. I. \& Varlamov, A. A. Theory of Fluctuations in Superconductors (Oxford Univ. Press, Oxford, 2005).

10. Shapiro, S. Josephson currents in superconducting tunneling: the effect of microwaves and other observations. Phys. Rev. Lett. 11, 80 (1963).

11. Kulik, I. O. Fluctuation conductivity of a tunnel contact at temperatures above critical. JETP Lett. 10, 313 (1969).

12. Hamilton, C. A. Josephson voltage standards. Rev. Sci. Instrum. 71, 3611 (2000).

13. De Gennes, P. G. Superconductivity of metals and alloys (Addison-Wesley, New York, 1989).

14. Martin, I. \& Balatsky, A. Probing pseudogap by Josephson tunneling. Phys. Rev. B 62, R6124(R) (2000).

15. Levchenko, A. Josephson current noise above $T_{c}$ in superconducting tunnel junctions. Phys. Rev. B 78, 104507 (2008).

\section{Acknowledgments}

This work was supported by the U.S. Department of Energy, Office of Science, Materials Sciences and Engineering Division. The work of A.S.M. was also supported by the Russian Foundation for Basic Research and the grant of the Russian Ministry of Science and Education (02.B.49.21.0003).

\section{Author contributions}

V.V. and A.M. conceived the work; A.G., A.M. and V.V. performed the calculations, A.G. carried out computational work; all authors discussed results and wrote the manuscript.

\section{Additional information}

Supplementary information accompanies this paper at http://www.nature.com/ scientificreports

Competing financial interests: The authors declare no competing financial interests.

How to cite this article: Galda, A., Mel'nikov, A.S. \& Vinokur, V.M. Resonant tunneling of fluctuation Cooper pairs. Sci. Rep. 5, 8315; DOI:10.1038/srep08315 (2015).

This work is licensed under a Creative Commons Attribution-NonCommercialNoDerivs 4.0 International License. The images or other third party material in this article are included in the article's Creative Commons license, unless indicated otherwise in the credit line; if the material is not included under the Creative Commons license, users will need to obtain permission from the license holder in order to reproduce the material. To view a copy of this license, visit http:// creativecommons.org/licenses/by-nc-nd/4.0/ 